\title{
Gravitational Shielding Effects in Gauge Theory of Gravity
}

\author{
Ning $\mathrm{Wu}^{*}$ \\ Institute of High Energy Physics, P.O.Box 918-1, Beijing 100039, P.R.China
}

October 30, 2018

PACS Numbers: 11.15.Ex, 04.60.-m, 11.15.-q.

Keywords: gravitational shielding effects, symmetry breaking, quantum gravity, gauge field.

\begin{abstract}
In 1992, E.E.Podkletnov and R.Nieminen find that, under certain conditions, ceramic superconductor with composite structure has revealed weak shielding properties against gravitational force. In classical Newton's theory of gravity and even in Einstein's general theory of gravity, there are no grounds of gravitational shielding effects. But in quantum gauge theory of gravity, the gravitational shielding effects can be explained in a simple and natural way. In quantum gauge theory of gravity, gravitational gauge interactions of complex scalar field can be formulated based on gauge principle. After spontaneous symmetry breaking, if the vacuum of the complex scalar field is not stable and uniform, there will be a mass term of gravitational gauge field. When gravitational gauge field propagates in this unstable vacuum of the complex scalar field, it will decays exponentially, which is the nature of gravitational shielding effects. The mechanism of gravitational shielding effects is studied in this paper, and some main properties of gravitational shielding effects are discussed.
\end{abstract}

\footnotetext{
*email address: wuning@mail.ihep.ac.cn
} 


\section{Introduction}

In 1992, E.E.Podkletnov and R.Nieminen find that, under certain conditions, ceramic superconductor with composite structure has revealed weak shielding properties against gravitational force[1, 2]. The gravitational shielding effects are hard to be understood in the traditional theory of gravity. The early attempt at a theoretical explanations were proposed by G.Modanese.[3] In classical Newton's theory of gravity and even in Einstein's general theory of gravity, there are no grounds of gravitational shielding effects. So, what is the mechanism of gravitational shielding effects? It is known that ordinary superconductor reveals no shielding properties against gravitational force, but in Podkletnov experiment, a specially prepared ceramic superconductor can reveal a weak gravitational shielding effect. Which properties of the specially prepared ceramic superconductor take a key role in making the superconductor reveal weak gravitational shielding effects?

It is well known that four kinds of fundamental interactions in nature can be well described by gauge field theory, and gauge principle is the common nature of all fundamental interactions. Great achievements of gauge field theory in describing strong, electromagnetic and weak interactions make us believe that the prospective quantum theory for gravity should be gauge field theory. Gauge treatment of gravity was suggested immediately after the gauge theory birth itself $[4,5,6,7,8,9]$. In 2001, a completely new quantum gauge theory of gravity is proposed by $\mathrm{N}$.Wu, which is the first perturbatively renormalizable quantum gravity in the 4-dimensional Minkowski space-time[10, 11]. In this approach, gravity, like electromagnetic interactions and strong interactions, is treated as a kind of physical interactions in flat space-time, not space-time geometry, so our basic physical space-time is always flat. In this new approach, the unification of fundamental interactions can be formulated in a simple and beautiful way[12, 13, 14]. If we use the mass generation mechanism which is proposed in literature [15], we can propose a new theory on gravity which contains massive graviton and the introduction of massive graviton does not affect the strict local gravitational gauge symmetry of the Lagrangian and does not affect the traditional long-range gravitational force[16]. The existence of massive graviton will help us to understand the possible origin of dark energy and dark matter in the Universe. In a recent work, it is found that the equation of motion of a classical mass point in gravitational field given by gauge theory of gravity is different from the geodesic equation of general relativity. Combining this equation of motion of a mass point with the perturbative solution of the field equation of gravitational gauge field, a correct theoretical expectation on classical tests of gravity can be obtained[17]. All these achievements make us believe that gauge theory of gravity is a possible prospective theory of quantum gravity. Based on this belief, we try to use gauge theory of gravity to explain quantum effects of gravitational interactions, such as phase effects in COW experiments[18, 19, 20], and we find that gauge theory of gravity gives out correct prediction on it[21]. In this paper, the gravitational shielding effects are studied in the framework of gauge theory of gravity. It is found that the gravitational shielding effects in Podkletnov experiments can be well explained by gauge theory of gravity in a simple and natural way. The reason 
why ordinary superconductor reveals no gravitational shielding effects but the specially prepared ceramic superconductor can reveal weak gravitational shielding effects is also discussed. The nature of gravitational shielding effects is studied.

\section{Gravitational Gauge Field}

First, for the sake of integrity, we give a simple introduction to gravitational gauge theory and introduce some notations which is used in this paper. Details on quantum gauge theory of gravity can be found in literatures [10,11]. According to discussions in literature [22], the symmetry for gravitational interactions can not be local Lorentz symmetry, and gauge principle tells us that the correct symmetry for gravitational interactions should be local translation symmetry, or gravitational gauge symmetry[10, 11]. In gauge theory of gravity, the most fundamental quantity is gravitational gauge field $C_{\mu}(x)$, which is the gauge potential corresponding to gravitational gauge symmetry. Gauge field $C_{\mu}(x)$ is a vector in the corresponding Lie algebra, which, for the sake of convenience, will be called gravitational Lie algebra in this paper. So $C_{\mu}(x)$ can expanded as

$$
C_{\mu}(x)=C_{\mu}^{\alpha}(x) \hat{P}_{\alpha}, \quad(\mu, \alpha=0,1,2,3)
$$

where $C_{\mu}^{\alpha}(x)$ is the component field and $\hat{P}_{\alpha}=-i \frac{\partial}{\partial x^{\alpha}}$ is the generator of gravitational gauge group, which satisfies

$$
\left[\begin{array}{lll}
\hat{P}_{\alpha}, & \hat{P}_{\beta}
\end{array}\right]=0 .
$$

Unlike the ordinary $S U(N)$ group, the commutability of the generators of the gravitational gauge group does not mean that the gravitational gauge group is an Abelian group. In fact, the gravitational gauge group is a non-Ablelian group[10, 11]. The gravitational gauge covariant derivative is given by

$$
D_{\mu}=\partial_{\mu}-i g C_{\mu}(x)=G_{\mu}^{\alpha} \partial_{\alpha}
$$

where $g$ is the gravitational coupling constant and $G$ is given by

$$
G=\left(G_{\mu}^{\alpha}\right)=\left(\delta_{\mu}^{\alpha}-g C_{\mu}^{\alpha}\right)
$$

Matrix $G$ is an important quantity in gauge theory of gravity. Its inverse matrix is denoted as $G^{-1}$

$$
G^{-1}=\frac{1}{I-g C}=\left(G_{\alpha}^{-1 \mu}\right)
$$

Using matrix $G$ and $G^{-1}$, we can define two important quantities

$$
\begin{gathered}
g^{\alpha \beta}=\eta^{\mu \nu} G_{\mu}^{\alpha} G_{\nu}^{\beta}, \\
g_{\alpha \beta}=\eta_{\mu \nu} G_{\alpha}^{-1 \mu} G_{\beta}^{-1 \nu} .
\end{gathered}
$$


The field strength of gravitational gauge field is defined by

$$
F_{\mu \nu} \triangleq \frac{1}{-i g}\left[D_{\mu} \quad, \quad D_{\nu}\right]
$$

Its explicit expression is

$$
F_{\mu \nu}(x)=\partial_{\mu} C_{\nu}(x)-\partial_{\nu} C_{\mu}(x)-i g C_{\mu}(x) C_{\nu}(x)+i g C_{\nu}(x) C_{\mu}(x) .
$$

$F_{\mu \nu}$ is also a vector in gravitational Lie algebra,

$$
F_{\mu \nu}(x)=F_{\mu \nu}^{\alpha}(x) \cdot \hat{P}_{\alpha}
$$

where

$$
F_{\mu \nu}^{\alpha}=\partial_{\mu} C_{\nu}^{\alpha}-\partial_{\nu} C_{\mu}^{\alpha}-g C_{\mu}^{\beta} \partial_{\beta} C_{\nu}^{\alpha}+g C_{\nu}^{\beta} \partial_{\beta} C_{\mu}^{\alpha} .
$$

\section{Gravitational Interactions of Complex Scalar Field}

In quantum theory, superconductive state, which is a macroscopic quantum state formed by Bose condensation of Cooper pairs in superconductivity, is described by a complex scalar field. So, in order to understand gravitational shielding effects in Podkletnov experiments, the gravitational interactions of complex scalar field should be studied first[23]. In principle, electromagnetic interactions are also involved in the Podkletnov experiments, but they do not take an essential role in the understanding of the gravitational shielding effects, so, for the sake of simplicity, the electromagnetic interactions are not considered in the present model.

In gauge theory of gravity, the lagrangian that describes the gravitational gauge interactions of complex scalar field is

$$
\mathcal{L}_{0}=-\eta^{\mu \nu}\left(D_{\mu} \phi\right)\left(D_{\nu} \phi\right)^{*}-V(\phi)-\frac{1}{4} \eta^{\mu \rho} \eta^{\nu \sigma} g_{\alpha \beta} F_{\mu \nu}^{\alpha} F_{\rho \sigma}^{\beta},
$$

where $V(\phi)$ is the potential of scalar field

$$
V(\phi)=\frac{1}{2} \mu^{2}|\phi|^{2}+\frac{\lambda}{4}|\phi|^{4}, \quad(\lambda>0) .
$$

The full Lagrangian of the system is

$$
\mathcal{L}=J(C) \cdot \mathcal{L}_{0},
$$


where

$$
J(C)=\sqrt{-\operatorname{det} g_{\alpha \beta}},
$$

and the corresponding action is

$$
S=\int \mathrm{d}^{4} x \mathcal{L}=\int \mathrm{d}^{4} x \quad \sqrt{-\operatorname{det} g_{\alpha \beta}} \cdot \mathcal{L}_{0}
$$

It can be strict proved that this action has strict local gravitational gauge symmetry, and therefore, this model is also perturbatively renormalizable.

When $\mu^{2}>0$, the minimum of the potential $V(\phi)$ is at origin $\phi=0$, so $\phi$ is the quantum field. From the above action, we can deduce the equation of motion of complex scalar field $\phi(x)$ and the field equations of gravitational gauge field $C_{\mu}^{\alpha}$. we can also calculate the inertial energy-momentum tensor of the system and the gravitational energy-momentum tensor of the system. Then, we can study gravitational interactions of complex scalar field $[10,11,23]$.

\section{Spontaneously Symmetry Breaking}

When $\mu^{2}<0$, the origin $\phi=0$ will become a local maximum and the symmetry of the system will be spontaneously broken. After symmetry breaking, the physical vacuum moves to $\phi_{0}$

$$
\left|\phi_{0}\right|=\sqrt{-\frac{\mu^{2}}{\lambda}}
$$

$\phi_{0}$ is the vacuum of the complex scalar field, generally speaking, it is required that $\phi_{0}$ is even distributed in microscopic scale, so that there is no quantum excitation, otherwise $\phi_{0}$ is not a classical state, but a quantum state. But on the other hand, $\left|\phi_{0}\right|^{2}$ represents Cooper pairs density in superconductor, and generally speaking, Cooper pairs density is not a constant in macroscopic scale, so $\phi_{0}$ can not be a constant in macroscopic scale. So, in this paper, our basic hypothesis on the physical vacuum $\phi_{0}$ is that it is a classical state, it is even distributed or a constant in microscopic scale and it is a function of space-time coordinates in macroscopic scale. In a word,

$$
\phi_{0}=\phi_{0}(x)
$$

$\phi_{0}$ is a classical state, it has no quantum excitation, though it is space-time dependent. After symmetry breaking, the scalar field becomes

$$
\phi(x)=\varphi(x)+\phi_{0} .
$$

$\varphi(x)$ is the quantum scalar field after symmetry breaking, which represents the small perturbations around physical vacuum $\phi_{0}$. 
After symmetry breaking, lagrangian becomes

$$
\begin{aligned}
\mathcal{L}_{0}= & -\eta^{\mu \nu}\left(D_{\mu} \varphi\right)\left(D_{\nu} \varphi\right)^{*}-\eta^{\mu \nu}\left(D_{\mu} \varphi\right)\left(D_{\nu} \phi_{0}\right)^{*}-\eta^{\mu \nu}\left(D_{\mu} \varphi\right)^{*}\left(D_{\nu} \phi_{0}\right) \\
& -\eta^{\mu \nu}\left(D_{\mu} \phi_{0}\right)\left(D_{\nu} \phi_{0}\right)^{*}-V(\varphi)-\frac{1}{4} \eta^{\mu \rho} \eta^{\nu \sigma} g_{\alpha \beta} F_{\mu \nu}^{\alpha} F_{\rho \sigma}^{\beta},
\end{aligned}
$$

where $V(\varphi)$ is the potential of scalar field

$$
V(\varphi)=\frac{\lambda}{4}|\varphi|^{4}+\frac{\lambda}{2}|\varphi|^{2}\left(\varphi^{*} \phi_{0}+\varphi \phi_{0}^{*}\right)-\frac{\mu^{2}}{2}|\varphi|^{2}+\frac{\lambda}{4}\left(\varphi^{* 2} \phi_{0}^{2}+\varphi^{2} \phi_{0}^{* 2}\right)-\frac{\mu^{4}}{4 \lambda} .
$$

There are two important properties which could be seen from the above lagrangian. Firstly, when the vacuum $\phi_{0}$ is space-time dependent, it can directly couple to quantum field $\varphi$. In other words, in the place where $\partial_{\mu} \phi_{0}$ does not vanish, vacuum state $\phi_{0}$ can directly excite quantum scalar state, so it can be considered to be an external source of scalar field. Secondly, when the vacuum $\phi_{0}$ is space-time dependent, it will couple to gravitational gauge field. But this interaction is completely in the classical level. In other words, it is also an external source of gravitational gauge field.

The field equation of gravitational gauge field in the superconductor is

$$
\partial_{\mu}\left(\eta^{\mu \rho} \eta^{\nu \sigma} g_{\alpha \beta} F_{\rho \sigma}^{\beta}\right)=-g T_{g \alpha}^{\nu},
$$

where $T_{g \alpha}^{\nu}$ is the gravitational energy-momentum tensor

$$
\begin{aligned}
T_{g \alpha}^{\nu}= & \eta^{\mu \nu}\left(D_{\mu} \varphi^{*}\right) \partial_{\alpha} \varphi+\eta^{\mu \nu}\left(D_{\mu} \phi_{0}^{*}\right) \partial_{\alpha} \varphi+\eta^{\mu \nu}\left(D_{\mu} \varphi\right) \partial_{\alpha} \varphi^{*}+\eta^{\mu \nu}\left(D_{\mu} \phi_{0}\right) \partial_{\alpha} \varphi^{*} \\
& +\eta^{\mu \nu}\left(D_{\mu} \varphi^{*}\right) \partial_{\alpha} \phi_{0}+\eta^{\mu \nu}\left(D_{\mu} \phi_{0}^{*}\right) \partial_{\alpha} \phi_{0}+\eta^{\mu \nu}\left(D_{\mu} \varphi\right) \partial_{\alpha} \phi_{0}^{*}+\eta^{\mu \nu}\left(D_{\mu} \phi_{0}\right) \partial_{\alpha} \phi_{0}^{*} \\
& -\eta^{\mu \rho} \eta^{\nu \sigma} g_{\beta \gamma} F_{\rho \sigma}^{\gamma} \partial_{\alpha} C_{\mu}^{\beta}+G_{\alpha}^{-1 \nu} \mathcal{L}_{0}+\eta^{\mu \rho} \eta^{\nu \sigma} g_{\alpha \beta} G_{\gamma}^{-1 \lambda}\left(D_{\mu} C_{\lambda}^{\gamma}\right) F_{\rho \sigma}^{\beta} \\
& -\frac{1}{2} \eta^{\mu \rho} \eta^{\lambda \sigma} g_{\alpha \beta} G_{\gamma}^{-1 \nu} F_{\mu \lambda}^{\beta} F_{\rho \sigma}^{\gamma}-\eta^{\lambda \rho} \eta^{\nu \sigma} \partial_{\mu}\left(g_{\alpha \beta} C_{\lambda}^{\mu} F_{\rho \sigma}^{\beta}\right) .
\end{aligned}
$$

In fact, when we deduce the field equation of gravitational gauge field from least action principle, the original field equation that we obtained is

$$
J(C) \cdot \partial_{\mu}\left(\eta^{\mu \rho} \eta^{\nu \sigma} g_{\alpha \beta} F_{\rho \sigma}^{\beta}\right)=-g J(C) T_{g \alpha}^{\nu} .
$$

Because $J(C)$ does not vanish, we can be eliminate it from the above equation to obtain the field equation eq.(4.6). Now, in order to obtain correct gravitational shielding effects, we need to start our discussions directly from eq.(4.8), which can be changed into another form

$$
\partial_{\mu}\left(\eta^{\mu \rho} \eta^{\nu \sigma} g_{\alpha \beta} F_{\rho \sigma}^{\beta}\right)=-g J(C) T_{g \alpha}^{\nu}+(1-J(C)) \partial_{\mu}\left(\eta^{\mu \rho} \eta^{\nu \sigma} g_{\alpha \beta} F_{\rho \sigma}^{\beta}\right) .
$$

The field equation of complex scalar field is

$$
\begin{aligned}
\eta^{\mu \nu} D_{\mu} D_{\nu} \varphi-\frac{\mu^{2}}{2} \varphi= & -\eta^{\lambda \nu}\left(\partial_{\mu} G_{\lambda}^{\mu}\right) D_{\nu} \varphi-\eta^{\mu \nu} D_{\mu} D_{\nu} \phi_{0} \\
& -\eta^{\lambda \nu}\left(\partial_{\mu} G_{\lambda}^{\mu}\right) D_{\nu} \phi_{0}-g \eta^{\mu \nu} G_{\alpha}^{-1 \kappa}\left(D_{\mu} C_{\kappa}^{\alpha}\right) D_{\nu} \varphi \\
& -g \eta^{\mu \nu} G_{\alpha}^{-1 \kappa}\left(D_{\mu} C_{\kappa}^{\alpha}\right) D_{\nu} \phi_{0}-\frac{\lambda}{2} \varphi^{*}\left(\varphi+\phi_{0}\right)^{2}-\frac{\lambda}{2} \varphi^{2} \phi_{0}^{*} .
\end{aligned}
$$


This field equation can be changed into another form

$$
\left(\eta^{\mu \nu} \partial_{\mu} \partial_{\nu}-\frac{\mu^{2}}{2}\right) \varphi=-\eta^{\mu \nu} D_{\mu} D_{\nu} \phi_{0}-\eta^{\lambda \nu}\left(\partial_{\mu} G_{\lambda}^{\mu}\right) D_{\nu} \phi_{0}-g \eta^{\mu \nu} G_{\alpha}^{-1 \kappa}\left(D_{\mu} C_{\kappa}^{\alpha}\right) D_{\nu} \phi_{0}+\cdots
$$

From the right hand side of the above equation, we can see that when the vacuum of the complex scalar field is not a constant, it will become source of quantum scalar field $\varphi$. In other words, inhomogeneous vacuum will excite quantum states.

\section{$5 \quad$ Gravitational Shielding Effect}

After some complicated calculations, the field equation (4.9) can be changed into the following form

$$
\partial_{\mu}\left(\eta^{\mu \rho} \eta^{\nu \sigma} g_{\alpha \beta} F_{\rho \sigma}^{\beta}\right)=-g N_{\alpha}^{\nu}+g^{2} M_{\alpha \beta}^{\nu \mu} C_{\mu}^{\beta}+\cdots
$$

where

$$
\begin{aligned}
N_{\alpha}^{\nu}= & \delta_{\alpha}^{\nu}\left[-\eta^{\mu \lambda}\left(\partial_{\mu} \phi_{0}\right)\left(\partial_{\lambda} \phi_{0}^{*}\right)+\frac{\mu^{4}}{4 \lambda}\right] \\
& +\eta^{\mu \nu}\left(\partial_{\mu} \phi_{0}\right)\left(\partial_{\alpha} \phi_{0}^{*}\right)+\eta^{\mu \nu}\left(\partial_{\mu} \phi_{0}^{*}\right)\left(\partial_{\alpha} \phi_{0}\right) \\
M_{\alpha \beta}^{\nu \mu}= & \left(\delta_{\alpha}^{\mu} \delta_{\beta}^{\nu}+\delta_{\beta}^{\mu} \delta_{\alpha}^{\nu}\right)\left[\eta^{\rho \lambda}\left(\partial_{\rho} \phi_{0}\right)\left(\partial_{\lambda} \phi_{0}^{*}\right)-\frac{\mu^{4}}{4 \lambda}\right] \\
+ & \left(\eta^{\mu \nu} \delta_{\beta}^{\lambda}-\eta^{\lambda \nu} \delta_{\beta}^{\mu}\right)\left[\left(\partial_{\lambda} \phi_{0}\right)\left(\partial_{\alpha} \phi_{0}^{*}\right)+\left(\partial_{\lambda} \phi_{0}^{*}\right)\left(\partial_{\alpha} \phi_{0}\right)\right] \\
- & \delta_{\alpha}^{\nu} \eta^{\mu \lambda}\left[\left(\partial_{\lambda} \phi_{0}\right)\left(\partial_{\beta} \phi_{0}^{*}\right)+\left(\partial_{\lambda} \phi_{0}^{*}\right)\left(\partial_{\beta} \phi_{0}\right)\right] .
\end{aligned}
$$

From above expressions, all terms in $N_{\alpha}^{\nu}$ and $M_{\alpha \beta}^{\nu \mu}$ are classical quantities, so $N_{\alpha}^{\nu}$ and $M_{\alpha \beta}^{\nu \mu}$ themselves are classical quantities. Therefore, the first two terms of the right hand side of eq.(5.1) do not represent interaction terms. The first term is the source of gravity in superconductor, and the second term is mass term of gravitational gauge field. In fact, from eq.(5.2), we can see that $N_{\alpha}^{\nu}$ is just the energy-momentum tensor of the vacuum of complex scalar field, which is the source of gravitational gauge field. But, because the coupling constant of gravitational interactions is extremely small and the total energy in a superconductor is finite, the magnitude of total gravitational gauge field generated by energy-momentum of the superconductor is many orders smaller than that of the gravitational gauge field generated by the earth. In experiments, we can neglect the gravity generated by the superconductor itself. So, $N_{\alpha}^{\nu}$ has no contribution to gravitational shielding effects. We neglect this term for the moment.

Let's turn to the second term of the right hand side of eq.(5.1). It is a mass term, but it is not a constant mass term, so it is a variable mass term or local mass term. At different positions, $M_{\alpha \beta}^{\nu \mu}$ has different values. It is known that, in vacuum space, graviton is massless and gravitational force are long range force. But in superconductor, gravitational gauge field obtain a small mass term, so in superconductor, gravitational force 
decreased exponentially, which is the nature of gravitational shielding effects. So, when gravitational field goes out the superconductor, it will becomes much weaker than it goes into the superconductor.

For Podkletnov experiments, the dominant component of earth's gravitational gauge field is $C_{0}^{0}$, which corresponds to classical Newton's gravity. So, in order to explain gravitational shielding effects quantitatively, we need to study the propagation of gravitational gauge field $C_{0}^{0}$ in superconductor. For earth's gravitational field $C_{\mu}^{\alpha}$, it is static, so we can set all time derivative of $C_{\mu}^{\alpha}$ to zero. From field equation (5.1), the following field equation can be obtained

$$
\nabla^{2} C_{0}^{0}=g^{2} M_{00}^{00} C_{0}^{0}+\cdots,
$$

where

$$
M_{00}^{00}=2\left|\nabla \phi_{0}\right|^{2}+2 V\left(\phi_{0}\right),
$$

and the contribution from $N_{0}^{0}$ is neglected. Now, the mass term becomes very simple form. In ordinary superconductor, the Cooper pair density is almost a constant and space gradient of $\phi_{0}$ is almost zero. In this case,

$$
M_{00}^{00}=2 V\left(\phi_{0}\right)=-\frac{\mu^{4}}{2 \lambda} .
$$

After omitting contribution from $N_{0}^{0}$, the field equation (5.4) becomes

$$
\nabla^{2} C_{0}^{0}=-\frac{g^{2} \mu^{4}}{2 \lambda} C_{0}^{0}
$$

Selecting spherical coordinate system. For the earth's gravitational field, $C_{0}^{0}$ is approximately a function of $r$ coordinate. In this case, the general solution of eq. (5.7) is

$$
C_{0}^{0}(r)=\frac{c_{1}}{r} \cos \left(\sqrt{\frac{g^{2} \mu^{4}}{2 \lambda}}\left(r-r_{0}\right)\right)+\frac{c_{2}}{r} \sin \left(\sqrt{\frac{g^{2} \mu^{4}}{2 \lambda}}\left(r-r_{0}\right)\right),
$$

where $r_{0}$ is the position of the lower surface of superconductor. Generally speaking, $\frac{g^{2} \mu^{4}}{2 \lambda}$ is a extremely small quantity(later, we will give a rough estimation on it), in a small region of ordinary superconductor, we have

$$
C_{0}^{0}(r) \approx \frac{c_{1}}{r} .
$$

That is, ordinary superconductor almost have no effects on earth's gravitational field, or it shows no gravitational shielding effects. But for Podkletnov experiment, $\phi_{0}$ is not a constant, $\left|\nabla \phi_{0}\right|^{2}$ is many orders larger than $2 V\left(\phi_{0}\right)$, so the dominant contribution of $M_{00}^{00}$ is $2\left|\nabla \phi_{0}\right|^{2}$ and it becomes a positive quantity. In this case, $M_{00}^{00}$ is not a constant. Denote $g^{2} M_{00}^{00}$ by $m_{g}^{2}$

$$
m_{g}^{2}=g^{2} M_{00}^{00}=2 g^{2}\left|\nabla \phi_{0}\right|^{2},
$$


and omit the influence from $N_{0}^{0}$, then the above field equation (5.4) becomes

$$
\nabla^{2} C_{0}^{0}=m_{g}^{2} C_{0}^{0}
$$

The general solution of the above equation is

$$
C_{0}^{0}(r)=c_{1} \frac{e^{-m_{g}\left(r-r_{0}\right)}}{r}+c_{2} \frac{e^{m_{g}\left(r-r_{0}\right)}}{r}
$$

In Podkletnov experiment, $c_{2}$ vanishes and earth's gravitational field decrease exponentially

$$
C_{0}^{0}(r)=c_{1} \frac{e^{-m_{g}\left(r-r_{0}\right)}}{r}
$$

That is, the earth's gravitational field decrease exponentially in inhomogeneous superconductor. Suppose that the thickness of the gravitational shielding region is $\Delta r_{0}$ and $m_{g}$ denotes the average value of the mass of graviton in this region, then the relative gravity loss in the upper surface of the superconducting disk is

$$
\varepsilon \approx m_{g} \Delta r_{0}
$$

\section{Podkletnov Experiments}

Now, let's get a rough estimation on gravitational shielding effects in Podkletnov experiment. Because Newton's gravitational constant is

$$
G_{N}=\frac{g^{2}}{4 \pi}
$$

So

$$
m_{g}^{2}=8 \pi G_{N}\left|\nabla \phi_{0}\right|^{2}
$$

Obviously, increase space gradient of $\phi_{0}$ will increase $m_{g}$, and therefore increase gravitational shielding effects. According to this spirit, the key structure of the ceramic superconductor disk in Podkletnov experiment is that is has three zones with different crystal structure and the gravitational shielding effects mainly come from the transition part of the disk, which is consists of randomly oriented grains with typical sizes between 5 and $15 \mu \mathrm{m}$. In the experiment, the upper layer of the disk is superconducting, while the lower layer is not. There is a transition region between the tow layers. The upper part of the transition region is partially in superconducting, some part of the transition region is critical, while the lower part of the transition region is not superconducting. Because of the granular structure of the lower part, the Cooper pair density is strongly inhomogeneous and the space gradient of $\phi_{0}$ will be large. Besides, the supercurrent is disturbed by a high frequency magnetic field, which will increase the space gradient of $\phi_{0}$. Because the supercurrent is only in the surface of superconductor, so the space gradient 
of $\phi_{0}$ is mainly determined by penetration depth $l_{0}$. Denote the maximum of $\phi_{0}$ as $\phi_{0 m}$, then we get the following estimation

$$
\left|\nabla \phi_{0}\right|^{2} \propto \frac{\left|\phi_{o m}\right|^{2}}{l_{0}^{2}} .
$$

For a complex scalar field, its probability is

$$
\rho(x)=-i\left(\dot{\phi}^{*} \phi-\phi^{*} \dot{\phi}\right)
$$

So, in superconductor, the Cooper pair density $\phi_{0}$ is

$$
\rho_{0}=-i\left(\dot{\phi}_{0}^{*} \phi_{0}-\phi_{0}^{*} \dot{\phi}_{0}\right)
$$

For an ordinary supercurrent, suppose that its lifetime is $T_{0}$. Generally speaking, the lifetime of supercurrent is several years long. Then we can get the following estimation

$$
\dot{\phi}_{0} \propto \frac{\phi_{0 m}}{T_{0}}
$$

Therefore,

$$
\begin{gathered}
\rho_{0} \propto \frac{\left|\phi_{0 m}\right|^{2}}{T_{0}}, \\
\left|\nabla \phi_{0}\right|^{2} \propto \frac{\rho_{0} T_{0}}{l_{0}^{2}} .
\end{gathered}
$$

The approximate magnitude of the mass $m_{g}$ of graviton in inhomogeneous supercurrent is

$$
m_{g}^{2} \approx 8 \pi G_{N} \frac{\rho_{0} T_{0}}{l_{0}^{2}} .
$$

For a rough estimation, taking that

$$
\begin{gathered}
\rho_{0} \sim 10^{32} \mathrm{~m}^{-3}, \\
T_{0} \sim 10 \text { year } \sim 3 \times 10^{8} \mathrm{~s},
\end{gathered}
$$

and for Podkletnov experiment,

$$
l_{0} \sim 10^{-8} m
$$

Using these rough estimations, we get

$$
m_{g} \sim 0.03 m^{-1} .
$$

If the thickness of the disk is about $0.01 \mathrm{~m}$, according to eq.(5.14), the relative gravity loss is about

$$
\varepsilon \sim 0.03 \%
$$


which quite close to the experimental value of $0.3-0.5 \%$. So, present model can semiquantitatively explain gravitational shielding effects in Podkletnov experiment.

So, now, we can understand the roles of some experimental techniques in Podkletnov experiment. The role of the upper layer of the disk is to keep high supercurrent density in the experiment. Gravitational shielding effects come from the transition region of the disk. The high frequency magnetic field is used to increase disturbance from outside of the disk and therefore to increase inhomogeneity of the suppercurrent and final gravitational shielding effects. Fast rotation of the disk is also favorable for gravitational shielding effects, for the effect of this rotation is that the direction of the disturbance from magnetic field changes with high speed, which will increase disturbance. We know that gravitational shielding effects come from inhomogeneity of supercurrents, so ordinary superconductor, which carries homogeneous supercurrents, has no gravitational shielding properties.

\section{Discussions}

In this paper, the gravitational shielding effects are discussed in the framework of quantum gauge theory of gravity. When gravitational field propagate in an inhomogeneous classical vacuum, graviton will obtain a small mass term. The gravitational force transmitted by massive graviton will decrease exponentially. So, in gauge theory of gravity, the nature of gravitational shielding effects is exponentially decreasing of gravitational field in inhomogeneous vacuum of scalar field after spontaneous symmetry breaking.

In a meaning, the present model only gives a qualitative explanation on gravitational shielding effects. Without a doubt, gravitational shielding effect found by E.Podkletnov and R.Nieminen is an important effect, which may cause a scientific evolution in the near future, or even change our life style. We can imagine that, in the near future, people can "walk" freely in the sky without a wing. In order to thoroughly understand and completely grasp the law of gravitational shielding effects, more experimental and theoretical study on it is needed. The present model can give some predictions on gravitational shielding effects:

1. In phase transition, ordinary supercurrent may reveal gravitational shielding effects, though it reveals no gravitational shielding effects in a stable superconducting state. This is because the supercurrent is strongly unstable and inhomogeneous in phase transition.

2. Inhomogeneous ordinary superfluid can also reveal gravitational shielding effects. In a word, under suitable conditions, any kind of matter which has superfluidity can reveal gravitational shielding effects. 
3. Quark-Gluon Plasma(QGP), which is a special state of matter, can also reveal gravitational shielding effects. In QGP, inhomogeneous QCD vacuum can make graviton obtain a small mass term. It is known that in the early stage, our universe is in QGP state. When our universe is in QGP state, long range gravity can be shielded by inhomogeneous vacuum. So, local fluctuation of matter can cause instability of gravity, which will enlarge fluctuations of matter, which is favorable for the formation of galaxy[24].

\section{References}

[1] E.Podkletnov and R.Nieminen, Physica C 203 (1992) 271.

[2] E. Podkletnov, Weak gravitational shielding properties of composite bulk $\mathrm{YBa}_{2} \mathrm{Cu}_{3} \mathrm{O}_{7-x}$ superconductor below $70 \mathrm{~K}$ under electro-magnetic field, report MSUchem 95, (cond-mat/9701074).

[3] G.Modanese, Europhys. Lett. 35 (1996) 413; G.Modanese, Phys. Rev. D 54 (1996) 5002; G.Modanese and J.Schnurer, Possible quantum gravity effects in a charged Bose condensate under variable e.m. field, report UTH-391/96, (gr-qc/9612022).

[4] R.Utiyama, Phys.Rev.101 (1956) 1597.

[5] A.Brodsky, D.Ivanenko and G. Sokolik, JETPH 41 (1961)1307; Acta Phys.Hung. 14 (1962) 21.

[6] T.W.Kibble, J.Math.Phys. 2 (1961) 212.

[7] D.Ivanenko and G.Sardanashvily, Phys.Rep. 94 (1983) 1.

[8] F.W.Hehl, J.D.McCrea, E.W.Mielke and Y.Ne'eman Phys.Rep. 258 (1995) 1-171

[9] F.W.Hehl, P. Von Der Heyde, G.D.Kerlick, J.M.Nester Rev.Mod.Phys. 48 (1976) $393-416$

[10] Ning Wu, Commun. Theor. Phys. (Beijing, China) 38 (2002): 151-156.

[11] Ning Wu, Gauge Theory of Gravity, talk given at Meeting of the Division of Particles and Fields of American Physical Society at the College of William \& Mary(DPF2002), May 24-28, 2002, Williamsburg, Virginia, USA; hep-th/0109145, hep-th/0207254.

[12] Ning Wu, Commun. Theor. Phys. (Beijing, China) 38 (2002): 322-326.

[13] Ning Wu, Commun. Theor. Phys. (Beijing, China) 38 (2002): 455-460.

[14] Ning Wu, Commun. Theor. Phys. (Beijing, China) 39 (2003): 561-568.

[15] Ning Wu, Commun.Theor.Phys., (Beijing, China) 36(2001) 169-172. 
[16] Ning Wu, Commun. Theor. Phys. (Beijing, China) 39 (2003): 671-674.

[17] Ning Wu, Classical Tests of Gauge Theory of Gravity (in preparation)

[18] R.Colella, A.W.Overhauser and S.A.Werner, Phys.Rev.Lett. (1975):1472-1474.

[19] S.A.Werner, R.Colella and A.W.Overhauser, Phys.Rev.Lett. (1975): 1053-1055.

[20] A.W.Overhauser and R.Colella, Phys.Rev.Lett. (1974):1237-1239.

[21] Ning Wu, Gravitational Phase Effects in Gauge Theory of Garvity (in preparation)

[22] Ning Wu, Tunan Ruan, Problems on Foundations of General Relativity, hepth/0303258.

[23] Ning Wu, Gravitational Gauge Interactions of scalar field, (has been accepted by Commun. Theor. Phys.)

[24] Ning Wu, Gravitational Shielding Effect of Quark Gluon Plasma in Gauge Theory of Garvity (in preparation) 